\title{
Proprićtés antimicrobiennes de trois plantes psammophiles du littoral togolais menacées de disparition
}

par l:yana Amana Kpemissi('), Komlan Batawila('), Kouami Kokou('), Kossi Koumaglo( $\left.{ }^{2}\right)$, (omlan de Souza $\left({ }^{3}\right)$, Philippe Bouchet( $\left.{ }^{4}\right)$ et Koffi Akpagana $\left({ }^{1}\right)$

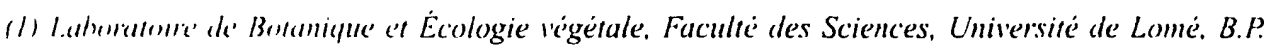
1515.6 .

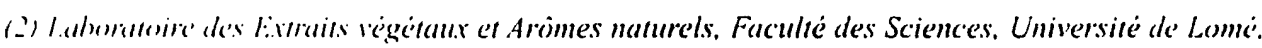

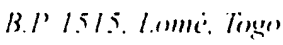

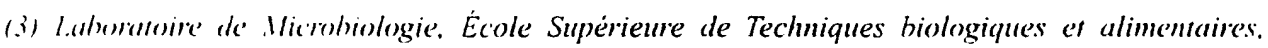
Liniversiti de Lome. B.P. 1515. Lomé. Togo

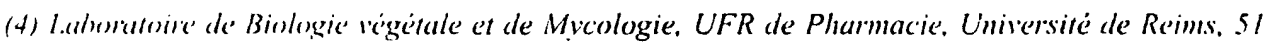

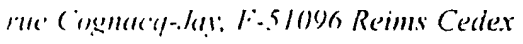

Résumé.- Les études pharmacologiques d'extraits de feuilles de trois espèces de plantes menacées de disparition du littoral du Togo (Conocarpus erectus $L$.. Dodonaea viscosa (L) Jacq. et Scaevola plumieri (L) Vahl.) ont permis de prouver leurs usages en thérapeutique traditionnelle. L'extrait hydro-éthanolique de ver leurs usages en therapeutique traditionnelle. Lextrait hydro-ethanolique de $D$. viscosa est très actif sur Bacillus subtilis, Pseudomonas aerugino-

sa. Staphyloccocus aureus et surtout Candida albicans avec une concentration minimale inhibitrice de $12.5 \mathrm{mg} / \mathrm{ml}$. Celui de $C$. erectus agit sur $S$. aureus et $P$ aeruginosa aiors qu'à la mème concentration celui de $S$. plumieri inhibe la croissance de $C$. albicans. Une étude chimique montre que les extraits renferment des tannins, des flavonoïdes et des saponines qui seraient à l'origine de leurs activités.

Mots-clés - Togo - espèces menacées de disparition - propriétés pharmacologiques - chimie.

Ahstract. - Pharmacological studies of leaves of three species threatened of the logolese coastal ecosystems (Conocarpus erectus $L$.. Dodonaea viscosa (L) Jacq. and Scaevola plumieri (L) Vahl.) prove their utilisation in the local pharma. copœia. Hydro-ethonolic extract of $D$. viscosa leaves is active on $B$. subtilis. $P$ copœia. Hydro-ethonolic extract of $D$. viscosa leaves is active on $B$. subtilis. $P$
aeruginosa, $S$. aureus and specially on C. albicans at $12.5 \mathrm{mg} / \mathrm{ml}$. The extract of aeruginosa, $S$. aureus and specially on $C$. albicans at $12.5 \mathrm{mg} / \mathrm{ml}$. The extract of $r i$ on $C$. albicans. These activities should be supported by tanins, flavonoids and saponins found in these extracts.

Ke'1-11ords: Togo - threatened species - pharmacological studies - chemistry. 


\section{INTRODUCTION}

I 'ulliciatien des plannes par l'I lomme est très anciemne. Maris, avec l'introduction du fer et de lien a dravers la mecanisalion de l'agriculture el la monoculture, on assiste de plus en plus it la degratlattion de la mature el la disparition des especes végétales. Ce phénomène s'accentue de nos jours avec la croissance démographicue el l'urbanisation. Les diverses comventions sur la nalture et la biodiversité (celle de Tumis en 1981 ainsi que celle de Rio (II 1992) mellent l'acecent sur le problème et préconisent des solutions appropriées.

In Alrique. la gestion de la diversité biologique, entreprise depuis la colonisation, a permis surtent la mise en place de programmes de conservation in sim à travers des aires prolegeces. Touteloris. la gestion de celles-ci n'a pas allénué les menaces qui pésent sur la llore

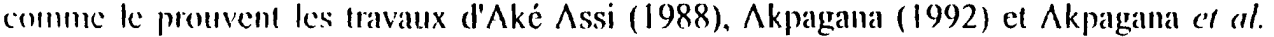
(1908). ('es études dressent un état des licux pour le littoral et la zone montagneuse du Togo. ( e's gones sont sujedtes à de fortes pressions anthropiques à travers la réduction des jacheres la récolle de bois-énergie, l'urbanisme, l'érosion côtière, ele. Des études prouvent (fuec parmi les espices ainsi menacées de disparition, plusieurs sont utilisces en thérapeulicjuc Mraditionnelle (Anomal \& Aké Assi, 1989); Iwu, 1993).

refle etude permet, en se fondame sur leurs usiges theraneuticunes locaux, de démontrer les proprietes antimicrobiemnes de trois espèces menacées de disparition de la llore logo-

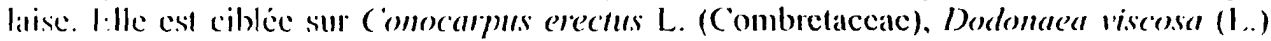

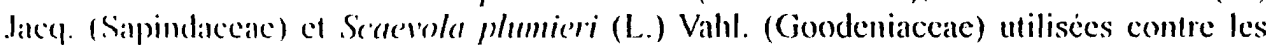
dermaltoses surtout. Toutes ces espèces appartiennent aux écosystèmes littoraux psammophiles oi clless sont très menacécs par la dégradation de l'environnement et par la coupe pour le bois de lew.

\section{MATIERILLS ET METTIOHISS}

\section{Nalciriel végétal}

Des cichantillons de plantes ont été récoltés en avril 1998. Leur identification a été faite suivant Bruncl ef al. (1984) et llutchinson et Dalziel (1954-1972) à l'herbier de l'Université de I nume oil des échrantillons de rélérence ont été déposés.

(. cretrus, connuc sous les noms vernaculaires "elaklogbe". "annckloghe". "alinigli" en cwe ou "apoussokpati" en mina, est un arbuste d'environ $3 \mathrm{~m}$ de hautcur. Les leuilles sont sourent glibres, allernes el cunéiformes à la base. Les inflorescences sont en tête splecricpese de 8 ; $10 \mathrm{~mm}$ de diamètre et les fruits, triangulaires, sont organisés en cônes moindes.

1. rivosor. lecalement appelée "xenu-attikesi" en éwé, est aussi un arbuste de 3 à 5 m de hatutertir dont les feruilles alternes sont simples, lancéolées et ponctuées de glandes résineuses. l'inflorescence est en grappe composée de fleurs pédicellées. Les fruits, ailés, contiemrent des graines ovö̈des.

S. phumicri est un arbuste buissommant à croissance sympodique et à ramification dichotomique. Il est localement commu en éwé sous les noms de "adikugbe" ou "apumeno". Les lieulles sont simples, charnues et alternes. Les fleurs, blanches, sont solitaires ou réunies en racènes. I.e fruit est une drupe bleu-noir à maturité. 


\section{B. Natériel microbien}

l.es souches microbiennes utilisées proviennent exclusivement de la collection du laboraltoire de Microbiologic de l'Écolc supérieure des techniques biologiques et alimentaires (I:STBA) de la même université. Il s'agit de quatre bactéries et d'une levure :

- bactéries ( iram- : Escherichia coli et Pseudomonas aeruginosa;

- bactiric (iram+: Bacillus subtilis et Staphylococcus aureus;

- levure: (amdida albicans.

\section{Néthodes}

\section{Preparation deses extratis}

Après síchage a température ambiante, $200 \mathrm{~g}$ de feuilles broyées de chaque plante sont extraits par macération hydro-éthanolique $\left(1 / 1\right.$, alcool à $\left.95^{\circ}\right)$ pendant cinq jours sous agitatton magnétique. L.e produit obtenu est filtré puis évaporé sous vide.

\section{Testr chimiques}

l.a determination des grands groupes chimiques (alcaloïdes, flavonoïdes, saponines et lannIns) de l'extrait de plante a été faite selon la technique de Harbone (1973).

\section{Pripuration des solutions d'extraits}

I.es solutions d'extrails sont obtenues par dilution d'une solution-mère de $100 \mathrm{mg} / \mathrm{ml}$ d'extrait brut de chacque plante. Les dilutions d'extrait de 1/2,1/4, 1/8, 1/16, 1/32 ont été essaycées sur les diflërents agents pathogènes testés.

\section{Príparation des suspensions de germes}

Des colonies de bactéries de 24 h et de C. albicans sont maintenues en culture respectivement sur milieu trypticase soja (TS) et bouillon de Sabouraud. Une colonie de chaque germe est prélevée puis triturée sur les parois internes d'un tube à essai contenant environ y) $\mathrm{ml}$ de bouillon Eugon stérile. Cinquante microlitres de la suspension homogénéisée sont prélevés et dilués dans $10 \mathrm{ml}$ de bouillon Eugon stérile. La solution obtenue constitue la suspension microbienne utilisée pour les essais.

\section{lest presomprif}

Dix microlitres de chaque suspension de germes sont mis en contact avec $0.5 \mathrm{ml}$ d'extraits non dilués des trois plantes dans des tubes à hémolyse et $0,5 \mathrm{ml}$ de bouillon Eugon et de bouillon Sabouraud, respectivement comme témoins pour les bactéries et la levure. L'enscmble des tubes (témoins et essais) est incubé à $37^{\circ} \mathrm{C}$ pendant $24 \mathrm{~h}$.

0.5 millilitre de chaque suspension microbienne incubée est étalé sur le milieu de culture dauns une boite de Petri et incubé à $37^{\circ} \mathrm{C}$ pendant $24 \mathrm{~h}$. À l'issue de l'incubation, on constate d'abord que le témoin a cultivé des colonies, en charge maximale. Puis, on compte le nombre de colonies dans les différents essais. L'absence totale ou partielle des colonies par rapport au témoin permet d'apprécier l'activité inhibitrice. En effet, s'il y a beaucoup de colonies, il y a survivance à l'extrait et donc peu d'inhibition. On peut alors evaluer le laux de survivance (TS) suivant la formule :

TS :- (nombre de colonies de l'essai/nombre de colonies du témoin) $\times 100$

(ette mesure permet de déduire le taux d'inhibition Ti par la relation $\mathrm{Ti}=100-\mathrm{TS}$. 


\section{RESULTATS ET DISCUSSION}

\section{A. Constiluants chimicues}

liétule chimique montre la présence de lamnins, de flavonoïdes et de saponines et l'al)sence d'alcaloüdes (Tableau 1). Les flavonoüdes et les saponines sont présentes dans les feuilles de (. erectus et $S$. plumieri, alors qu'ils sont absents dans celles de $D$. viscosa.

Tableau 1.- Conslituants chimiques des extraits éthanoliques de $C$. erectus, $D$. viscosa et $S$. plumieri.

Table I.- Chemical contituents of $C$. erectus, $D$. viscosa and $S$. plumieri ethanolic leaves extract.

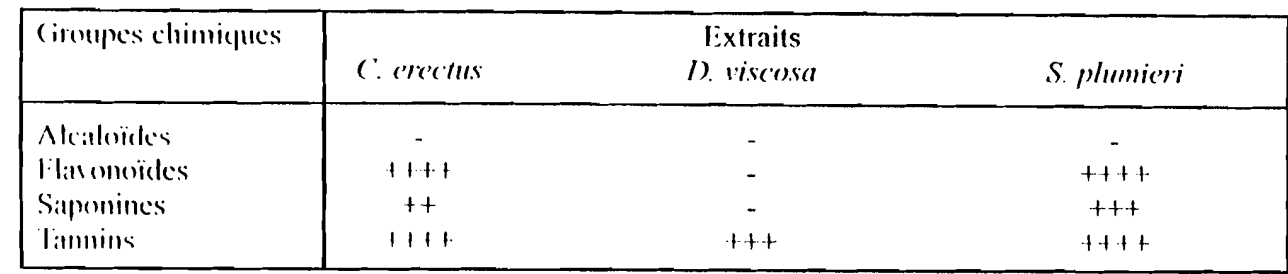

\section{B. Activité antimicrobienne des extraits}

I.es resultalts des tests antimicrobiens som résumés dans le tablcau II qui montre gue les germes sont inhlibes de dillërentes manières aux dillèrentes concentrations. $E$. coli est inhibe a 40$)^{\prime \prime}$ a $50 \mathrm{mg} / \mathrm{ml}$ d'extrait, alors que les autres germes le sont à $99 \%$ ( $S$. aureus. $P$.

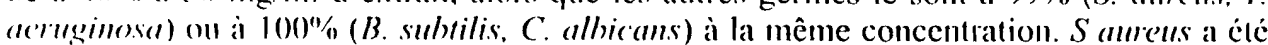
partiellement inhihe aux différentes concentrations utilisées.

Tableau II.- Pourcentage d'inhibition des extraits de feuilles de $C$. erectus. Table II.- Inhibition percentage of $C$. erectus leaves extract.

\begin{tabular}{|c|c|c|c|c|c|c|}
\hline \multirow[b]{2}{*}{ (iermess } & \multicolumn{6}{|c|}{ Activité à différentes concentrations ( $\mathrm{mg} / \mathrm{ml})$} \\
\hline & 100 & 50 & 25 & 12.5 & 6.25 & 3,125 \\
\hline S. anrins & 99,99 & 99.99 & 99,94 & 99,98 & () & 0 \\
\hline licoli & 100 & 40 & 0 & 0 & () & 0) \\
\hline l? arerginosu & 100 & 99.99 & 99,98 & 99.98 & 0 & 0 \\
\hline B. suhilis: & 100 & 100 & 99,99 & 0 & () & () \\
\hline c allicams & 100 & 100 & 99,99 & 20 & () & ) \\
\hline
\end{tabular}

L.ess extraits totaux de $C$. erectus ont une activité germicide sur les bactéries et la levure retentes. E. coli possède l'aire de survivance la plus étendue et demeure, de ce fait, l'espèce la plus résistante. En effet, à la concentration de $50 \mathrm{mg} / \mathrm{ml}$, son taux de survivance est (le $6(1) \%$ alors qu à $12,5 \mathrm{mg} / \mathrm{ml}$, ce taux est nul pour $S$. aureus et $P$. aernginosa. Ces germes sont de ce lait les moins résistants à l'extrait total des feuilles de $C$. erectns. Certains individus de ( $C$. allicinns survivent dès que la concentration de l'extrait baisse à $12,5 \mathrm{mg} / \mathrm{ml}$. Son taux de survivance qui était nul à $25 \mathrm{mg} / \mathrm{ml}$ passe à $80 \%$ à $12,5 \mathrm{mg} / \mathrm{ml}$. La représenlation sous forme d'histogramme des résultats de l'action des différentes concentrations de l'extrail des leuilles de cette plante sur les microbes testés est domée par la figure l. 


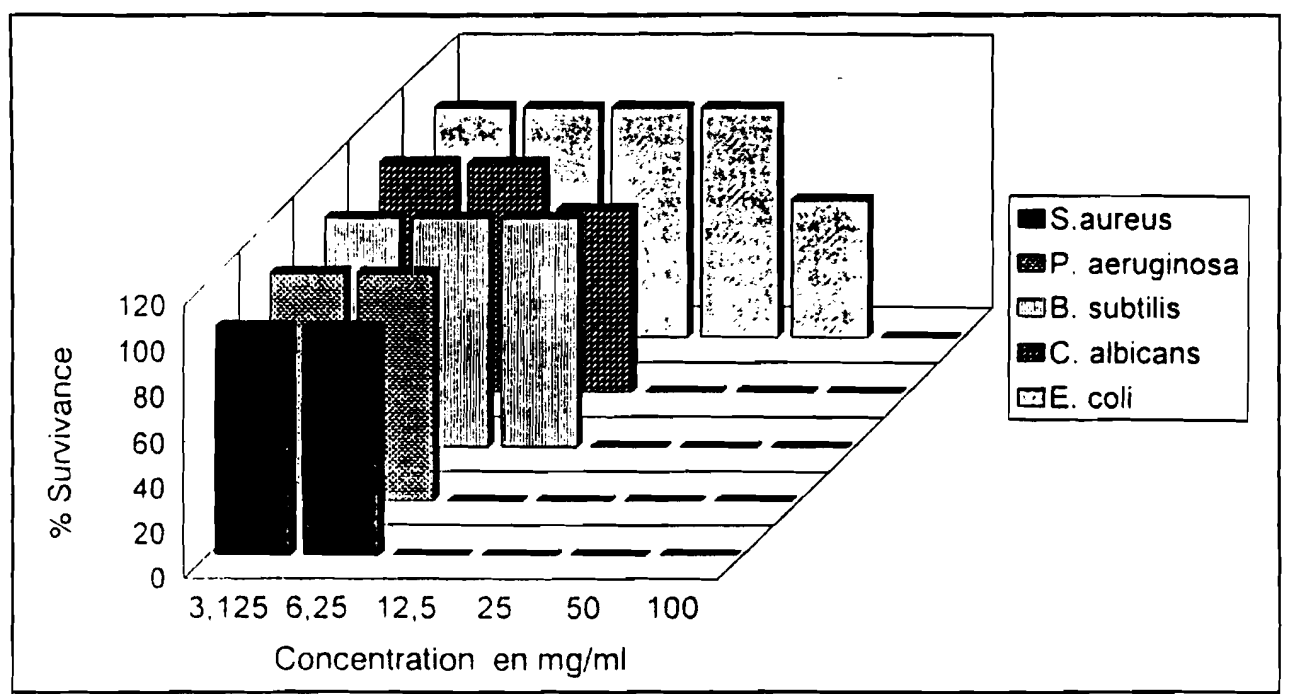

Fig. 1.- Pourcentage de survivance des différents germes à l'extrait de feuilles de C. erectus.

Fig. 1.- Survivance percentage of different germs to $C$. erectus leaves extracts.

I 'extrait total de lemilles de c.. erectus a cu des activités différentes sur les deux bactéries ( iram-. I:n elliel. l: colli s'est montré très tôt résistante à l'action de l'extrait a la concentration de 50 ) méml. alors que P. aernginosa est inhibé à $99 \%$ à la même concentration. À la concentration de $25 \mathrm{mg} / \mathrm{ml}$, tous les individus de $E$. coli survivent alors que ceux de $P$. "rrugimens som inhlibis à $99 \%$. Celte inhibition demeure élevée, même à la concentration de $12.5 \mathrm{mg} \mathrm{ml}$. Sur les bactéries Gram+. l'activité des extraits varie également selon les difFërentes conncentrations. Alors que $S$. aureus a été partiellement inhibé par la solution de l'extrait hrut dans notre manipulation, il est resté paradoxalement inhibé jusqu'à 99\% lorselue la concentration a baissé a $12.5 \mathrm{mg} / \mathrm{ml}$. à celte concentration. tous les individus de B. vhrilis survivent. De tous les germes utilisés. E. coli semble le plus résistant : il survit il $(0)$ ") a i une concentratton d'extrait de $50 \mathrm{mg} / \mathrm{ml}$. Certains auteurs tels que Berhaut (1967) estiment yue l'icorce de cette espèce contiendrait des tanins. Par ailleurs. Baba-Moussa (1909) ainsi que Balbal-Moussa ét al. (1999) ont montré que l'activité antifongique de certaines (ombretaceace ouest-alricaines serait liće à des tanins et à des flavonoüdes.

1.'acurile de 1). riscosa a dommé des résultats qui sont présentés dans le tableau III ciapris. 11 montre que cet extrait est efficace jusqu'à $25 \mathrm{mg} / \mathrm{ml}$. À cette même concentration. l: coli est resistiant tandis que $C$. albicans et $S$. aureus demeurent les moins résistants. $\dot{A}$ la concemtration $6.25 \mathrm{mg} / \mathrm{ml}$, ces demiers sont inhibés à $99 \%$. $\dot{A} 3,125 \mathrm{mg} / \mathrm{ml}$. C. albican.s reste inhile ai $(9) "$. lin terme de survivance, ces résultats sont exprimés par la figure 2 . Cet

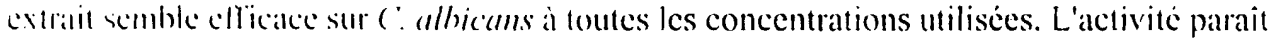
semblable sur les backeries (irann+ S. aureus et $B$. subtilis. Elle diffëre lorsque la concentration de l'exirail cist de $0.25 \mathrm{mg} / \mathrm{ml}$. Sur les bactéries Gram-, l'activité germicide parait signilicattice. $A 25 \mathrm{mg}$ ml. le laux de survie est nul pour P. aeringinosa. Il est de $30 \%$ pour 
Tableau III.- Pourcentage d'inhibition des extraits de feuilles de D. viscosa. Table III.- Inhibition percentage of $D$. viscosa leaves extract.

\begin{tabular}{|c|c|c|c|c|c|c|}
\hline \multirow[b]{2}{*}{ Giermes } & \multicolumn{6}{|c|}{ Activité à dillërentes concentrations (mg/ml) } \\
\hline & 100 & 50 & 25 & 12.5 & 6.25 & 3.125 \\
\hline S. allmems & 100 & 100 & 99,85 & 80 & 99,90 & () \\
\hline l: ioli & 100 & 99,99 & 70 & 0 & 0 & () \\
\hline l' acrergimosed & 100 & 99,99 & 99,99 & 97.60 & () & 0 \\
\hline B. sulvilis & 100 & 99,99 & 99.99 & 99,99 & 0 & 0 \\
\hline ( : alhicams & 100 & 100 & 99,99 & 99,99 & 99.98 & 99.91 \\
\hline
\end{tabular}

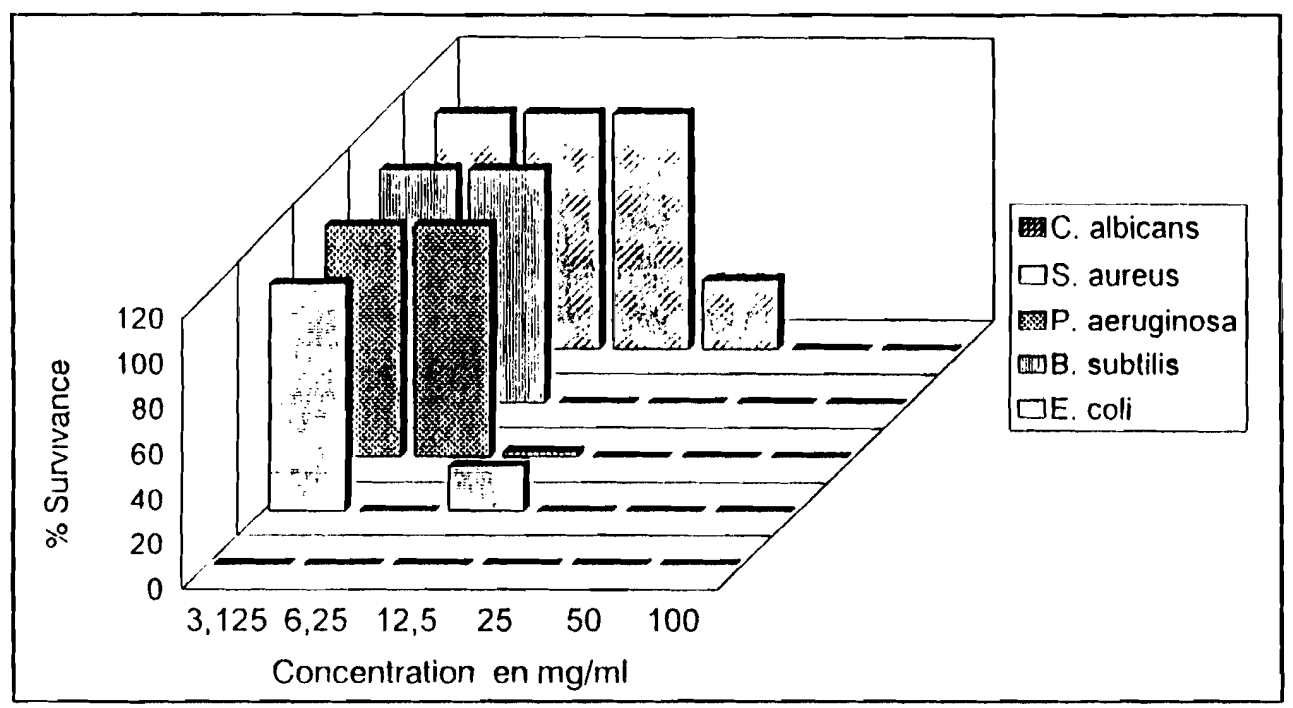

Fig. 2.- Pourcentage de survivance des différents germes à l'extrait de feuilles de $D$. viscosa.

Fig. 2.- Survivance percentage of different germs to $D$. viscosa leaves extracts.

$E$ coli ; alors qu'à $12.5 \mathrm{mg} / \mathrm{ml}$, la survivance est totale pour $E$. coli, elle est pratiquement infericure à $3 \%$ pour $P$. aerluginosa.

Des travaux antéricurs ont révélé que les feuilles de D. viscosa contenaient des huiles essenlielles, des llavonoüdes, des diterpenoïdes, des stérols et des sapogénines (Belachew, 1993 : Alımad ef al., 1987 ; Alımad el al., 1994; Mata et al., 1991, cités par Baba Moussa. 1999). (es mêmes auteurs montrent que certains de ces composés secondaires sont ellicaces sur les bactéries (irant (B. surilis, $B$. hronchiseptica et $S$. amedes). les bactéries (iram- (l. coli et l? aeringinosa).

$\dot{\Lambda}$ diflerentes concentrations, l'aclivite d'extrait de leuilles de S. phumieri a donne les resulaals presentés dams le tableau IV. 
Tableau IV.- Pourcentage d'inhibition des extraits de feuilles de S. plumieri. Table IV.- Inhibition percentage of S. plumieri leaves extract.

\begin{tabular}{|c|c|c|c|c|c|c|}
\hline \multirow[b]{2}{*}{ (icrmes } & \multicolumn{6}{|c|}{ Activité à différentes concentrations $(\mathrm{mg} / \mathrm{ml})$} \\
\hline & 100 & 50 & 25 & 12,5 & 6.25 & 3,125 \\
\hline S. allrems & 99.96 & 99.96 & 99,86 & 20 & 0 & 0 \\
\hline$E(c) / i$ & 100 & 98.12 & 40 & 0 & () & 0 \\
\hline l' acrugrimosu & 100 & 100 & 99,99 & 20 & 0 & () \\
\hline B. suhilis & 100 & 99.99 & 99.98 & 0 & 0 & 0 \\
\hline (' allhicams & 100 & 99.99 & 99,99 & 98.97 & 98.00 & 40) \\
\hline
\end{tabular}

C's résultats montremt que les extraits de feuilles de cette espèce ont une activité inhibitrice sur lous les germes utilisés. Cependant, $E$. coli se révèle résistante car son taux d'inhibitun n'est cute de $40 \%$ à la concentration de $25 \mathrm{mg} / \mathrm{ml}$ alors que tous les autres germes somt inlubis a $94 \%$ a cette même concentration. $C$. alhicans demeure l'espèce la moins resistante i l'extrait. Il convient de souligner que $S$. aureus n'a été que partiellement inhibi au cours des différents essais. La figure 3 exprime, en terme de survivance, ces résullats. L.'extrail lolal de cettc espèce est d'une faible efficacité tant sur les bactéries Gram(ju sur les bactiries (jam+. L'activité est semblable tant pour $S$. aureus que pour $P$. aertuginosa. ()n peut laire le mème rapprochement entre $E$. coli et $B$. subtilis. Néanmoins, $E$. coli est plus risistante à l'action de l'extrait de cette plante. Vis-à-vis de $C$. albicans, cet extrait est tres cllïacace. En effè, à la concentration de $6,25 \mathrm{mg} / \mathrm{ml}, 98 \%$ des individus tes-

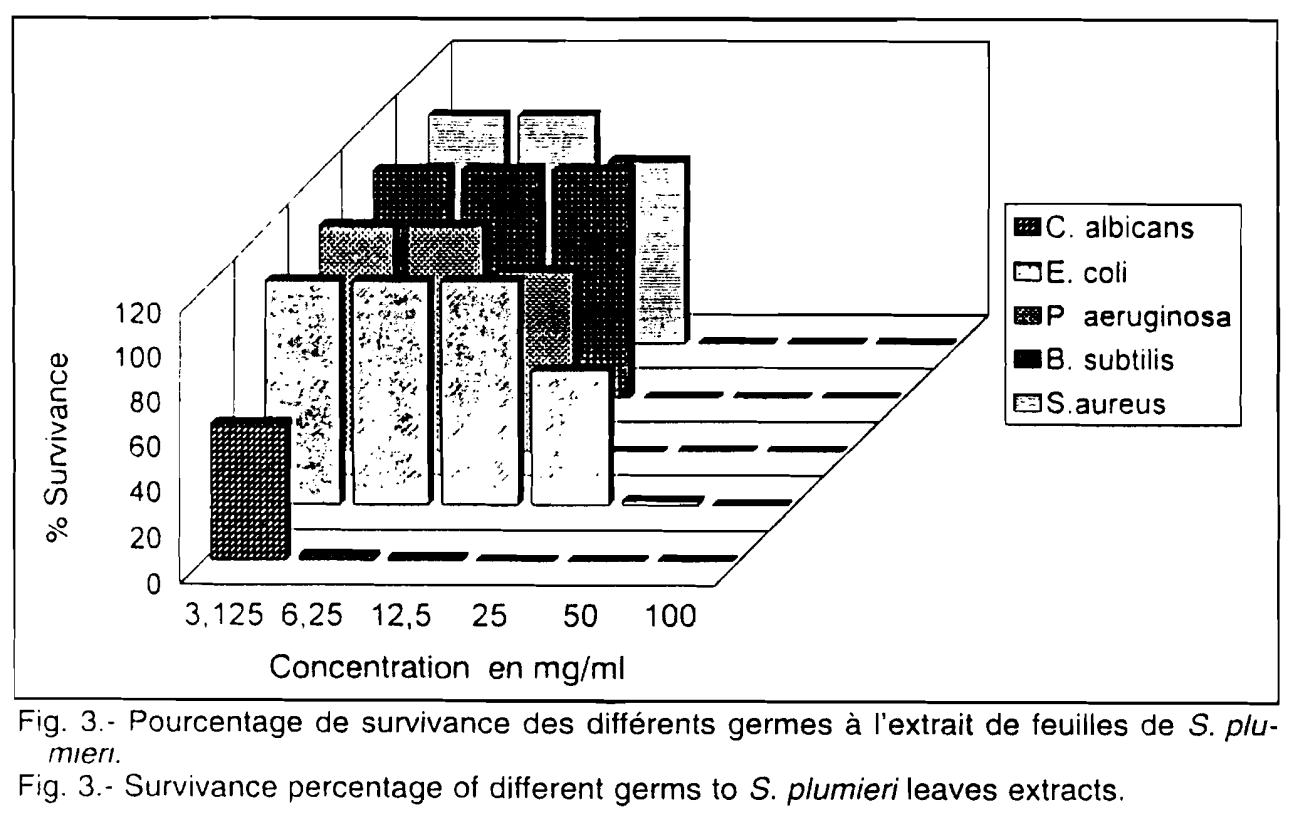

Fig. 3.- Survivance percentage of different germs to $S$. plumieri leaves extracts. 
Tableau V.- Pourcentage d'inhibition comparé des différents extraits à $12,5 \mathrm{mg} / \mathrm{ml}$. Table V.- Compared Inhibition percentage of different extracts at $12.5 \mathrm{mg} / \mathrm{ml}$.

\begin{tabular}{|c|c|c|c|}
\hline (icrmes: & C. corcentss & $\begin{array}{l}\text { cnlage d'imh } \\
\text { D. viscosa }\end{array}$ & S. plumieri \\
\hline S. anrerns & 99,98 & 99.90 & 20 \\
\hline B. suhtrilis & 0 & 99,99 & 0 \\
\hline$l$ coli & 0 & 0 & 0 \\
\hline I' areruginosat & 90.98 & 97,60 & 20 \\
\hline ( allicalls. & 20 & 99,99 & 98,97 \\
\hline
\end{tabular}

tes som inhibes. Il apparait done que l'extrait de feuilles de $S$. plumieri présente un intérêt. a de lables concentmations, sur cetle levure.

Des itudes antéricures, notamment celles de Skaltsounis, Tellequin et al. (1989) et de Skalısomis. Sbahi ct al. (1989) sur d'autres espèces du genre Scaevola indiquent la présence d'iridöles (le loganoside, l'acide loganique, le sylvestroside III, l'acétal méthylicue du sylicstroside III, le cantleyoside, l'acétal méthylique du cantleyoside et le scaevoloside) qui seraient a la base de celle activité.

Syuthéticuement. il est possible de rassembler pour établir un tableau comparatir de l'activ ite des differents extraits a $12.5 \mathrm{mg} / \mathrm{ml}$ sous la forme du tableau $V$. 11 montre que $S$. anrens est inhibe par les extraits de $C$. erectus et $D$. viscosa et dans une moindre mesure par l'extrait de S. plumieri. B. suhtrilis est inhibé seulement par celui de D. viscoser. E. coli est insensible a tous les extraits des trois plantes. P. aernginosa est inhibé à la même échelke que S. anrens. (. alhicans est inhibé par les extraits de D. viscossa et $S$. phumieri et accessoirement par l'extrait de C. ereectus.

\section{CONCLUSION}

Chacunc des trois plantes étudiées a montré une activité antibactérienne el antilongigue variable sclon la concentration des extraits. II ressort de celte étude que :

- S. anrous, P. acruginosa et C. albicans ont été les germes les plus sensibles ; rappelons qui $P$. aeruginosa est un germe génćralement très résistant ;

- l'extrait de $D$. viscosa a été le plus efficace ; il présente donc plus d'intérêt du point de vac de l'activité antimicrobienne; l'activité de cette espèce est suivie de celles de $C$. erectris et de S. plumiceri.

L'extrail de D. viscosel élant très actif sur $C$. albicans, il convient de lui prêter une altenlion parliculicre car cette activité constitue une piste sérieuse de recherche contre cette souche fongique pathogènc, souvent difficile à combattre.

Il résulte de tout ce qui précède que ces trois espèces végétales, actuellement menacées de disparition, doivent être conservées. Cette conservation passe par une gestion duable à lavers :

- la multiplication par germination de graine ou par bouturage : 
- la constitution de réserves de plants en vue de l'introduction d'individus au jardin botanicue et dans leurs sites naturels dans une approche participative integrant les populations locales :

- la poursuite des études pharmacologiques en mettant l'accent sur la chimie afin d'identifier la fraction active.

\section{BHIBL.IC)( IRAPHIIE}

Ahmad 1. M. Ahmad \& A. Ahmad. 1994. Antimicrobral activity of Dodonaea viscosa oil. Fitoterapia, 65 (2). $167 \cdot 168$

Ahmad V.U., I. Fatima \& A. Fatima, 1987.- The sapogenins from Oodonaea viscosa. Fitoterapia. 58 (5). 361 . 362.

Akpagana K. 1992. Quelques espèces rares ou mena cées de disparition du Togo. 1 - Le cordon littoral. Ann. Univ. Bénin, sér. Sci. 10. 33-36.

Akpagana K., J.T. Arnason. A. Akoègninou \& P Bouchet. 1998. La disparition des espèces vegetales en Afrique tropicale. Cas du Togo et du Bénin en Alrique de l'Ouest. Le Monde des Plantes, 463. 18-20.

Anoma G. \& L. Aké Assi. 1989.- Flore de Côte d'lvoire. Disparition de nombreuses espèces due à la destruc. tion inconsidérée de l'espace naturel, le cas d'un plante médicinale : Monanthotaxis capea (E G \& A. plante medicin : Monanhoris capea (E. G. \& A. Camus) Verdc. (Annonaceae). Bull. Méd. Trad.

Baba-Moussa F 1999. Recherches sur les propnetes antifongiques de plantes utilisees en médecine trad ionnelle au Bénin et au Togo. Thèse doctorat d'Élal. Universitè de Reims. $157 \mathrm{p}$

Baba-Moussa F.. K. Akpagana \& P Bouchet. 1999 Antifungical activities of seven West Alrican Combretaceae used in traditional medicine. Joumal or Ethnopharmacology. 66. 335.338.
Belachew D. 1993. Elhiopian traditional herbal drugs. Part II - Antimicrobial aclivity of 63 medicinal plants. Joumal of Ethnophamacology. 39. 129-139.

Brunel J.F. P. Hiekpo \& H. Scholz. 1984. Flore analytlque du Togo: Phanerogames. GTZ éd.. Eschborn. $751 \mathrm{p}$

Harbone J.B.. 1973.- Phytochemical methods. Chapman and Hall eds., New York, $354 \mathrm{p}$

Hutchinson J. \& J.M Dalziel. 1954-1972. Fiora of West Tropical Africa, 2nd ed. revised by Keay \& Hepper, 3

Iwu M. M.. 1993.- Handbook of African Medicinal Plants. CAC Press, Inc. Boca Raton, Ann Arbor, London. Tokyo, 1.7

Mala P.. J.L.Contreras. D.Crisanto. R Pereda-Miranda \& $P$ Castaneda. 1991. Chemical studies on Mexican plants used in traditionnal medicine. XVIII - New secondary metabolites from Dodonaea viscosa. Joumal of Natural Products. 54 (3). 913.917.

Skaltsounis A.L. S Sbahi. C. Demetzos \& J Pousset 1989. Plantes de Nouvelle-Calédonie. Iridoides de Scaevola montana Labill. Ann. Pharn. Fr. 47 (4). 249.254

Skallsounis A.L.. F Tellequin. M. Koch. J. Pousset \& G. Chauvière. 1989.- Iridoids from Scaevola racemifera. Planta Medica, 55. 191-192. 